\title{
Structure and function of the initially transcribing RNA polymerase II-TFIIB complex
}

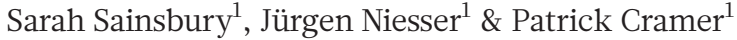

\begin{abstract}
The general transcription factor (TF) IIB is required for RNA polymerase (Pol) II initiation and extends with its B-reader element into the Pol II active centre cleft. Low-resolution structures of the Pol IITFIIB complex ${ }^{1,2}$ indicated how TFIIB functions in DNA recruitment, but they lacked nucleic acids and half of the B-reader, leaving other TFIIB functions ${ }^{3,4}$ enigmatic. Here we report crystal structures of the Pol II-TFIIB complex from the yeast Saccharomyces cerevisiae at $3.4 \AA$ resolution and of an initially transcribing complex that additionally contains the DNA template and a 6-nucleotide RNA product. The structures reveal the entire B-reader and proteinnucleic acid interactions, and together with functional data lead to a more complete understanding of transcription initiation. TFIIB partially closes the polymerase cleft to position DNA and assist in its opening. The B-reader does not reach the active site but binds the DNA template strand upstream to assist in the recognition of the initiator sequence and in positioning the transcription start site. TFIIB rearranges active-site residues, induces binding of the catalytic metal ion $B$, and stimulates initial RNA synthesis allosterically. TFIIB then prevents the emerging DNA-RNA hybrid duplex from tilting, which would impair RNA synthesis. When the RNA grows beyond 6 nucleotides, it is separated from DNA and is directed to its exit tunnel by the B-reader loop. Once the RNA grows to 12-13 nucleotides, it clashes with TFIIB, triggering TFIIB displacement and elongation complex formation. Similar mechanisms may underlie all cellular transcription because all eukaryotic and archaeal RNA polymerases use TFIIB-like factors ${ }^{5}$, and the bacterial initiation factor sigma has TFIIB-like topology $y^{1,2}$ and contains the loop region 3.2 that resembles the B-reader loop in location, charge and function ${ }^{6-8}$. TFIIB and its counterparts may thus account for the two fundamental properties that distinguish RNA from DNA polymerases: primer-independent chain initiation and product separation from the template.
\end{abstract}

Our previous X-ray analysis of the Pol II-TFIIB complex at $4.3 \AA$ resolution provided a partial backbone model of TFIIB $^{1}$. To obtain a complete and atomic structure, we co-crystallized Pol II with a TFIIB variant lacking the mobile amino-terminal tail and carboxy-terminal cyclin fold (Methods, Supplementary Table and Supplementary Fig. 1). The resulting Pol II-TFIIB structure at $3.4 \AA$ resolution provides details of the interactions of the four TFIIB domains with Pol II: the B-ribbon with the dock, the B-core N-terminal cyclin fold with the wall, the B-reader helix with the RNA exit tunnel, and the B-linker helix with the coiled-coil of the clamp (Fig. 1).

The structure reveals the entire course of the TFIIB polypeptide chain through the Pol II cleft, including the previously lacking B-reader loop (residues 67-79) and a new 'B-reader strand' (residues 80-83). The B-reader loop does not reach the active site, but instead interacts with the Pol II rudder and fork loop 1 to seal part of the cleft. The B-reader strand adds to the two-stranded polymerase lid (Fig. 1a, c), positioning the B-reader and explaining why the lid is essential for transcription ${ }^{9}$. There is no evidence for a B-finger ${ }^{2}$.

TFIIB binding induces changes in Pol II domains (Fig. 2). The lobe, protrusion and wall rotate slightly, partially closing the cleft.
The previously mobile ${ }^{10}$ tip of the protrusion forms a $\beta$-sheet and an ordered loop $(\alpha 11-\alpha 12)$ that is required for initiation complex stability $^{11}$ (Fig. 1d). To induce these changes, the B-ribbon may bind the dock, causing the wall to rotate, which then enables the B-core to bind the wall and protrusion (Figs $1 \mathrm{~d}$ and $2 \mathrm{a}$ ). TFIIF also binds the lobe and protrusion ${ }^{12,13}$, and stabilizes TFIIB on Pol $\mathrm{II}^{14}$, in particular binding of the B-core to the wall ${ }^{15}$. These changes contribute to TFIIB function, because mutations in the lobe and protrusion (Supplementary Fig. 2) suppress a TFIIB mutation that causes shifts in the transcription start site (TSS) $)^{16}$.

The structural changes extend to the polymerase active site, where difference electron density revealed two metal ions with similar occupancy (Fig. 2b). This was consistent with a two-metal ion catalytic mechanism ${ }^{17,18}$, but was unexpected because free Pol II only binds metal A, whereas metal B was observed previously only when a nucleoside triphosphate (NTP) was present $t^{19,20}$. The two metal ions are bridged by a rotated aspartate D481 side chain (Fig. 2b), which is also observed in structures of NTP-containing elongation complexes of Pol $\mathrm{II}^{20}$ and bacterial polymerase ${ }^{21}$. The rearrangement abolishes a contact between metal A and residue D483, and re-orientates the backbone carbonyl in the neighbouring Rpb2 residue D837 (Fig. 2b).

This indicates that TFIIB induces active-site rearrangements that increase the affinity for metal B and stimulate catalysis. Consistent with this, the B-reader of the archaeal TFIIB homologue stimulates transcription $^{22}$; truncation of the B-reader loop in human TFIIB impairs extension of a RNA dinucleotide ${ }^{23}$; and the presence of TFIIB and TBP stimulates transcription initiation from pre-opened $\mathrm{DNA}^{15,24}$. We find here that TFIIB alone stimulates de novo RNA synthesis by Pol II at low or near-physiological NTP concentrations (Fig. 2c and Supplementary Fig. 3). Thus, TFIIB stimulates RNA chain initiation without contacting the catalytic site, by activating Pol II allosterically.

We also solved the structure of an initially transcribing Pol II-TFIIB complex (ITC) by soaking into the crystals a HIS4 promoter-based scaffold with a downstream DNA duplex and a 6-base-pair (bp) DNARNA hybrid. Electron density was observed for the entire RNA, 7 bp of downstream DNA, and the DNA template strand until position -8 upstream of the NTP site +1 . The structure at $3.6 \AA$ resolution (Fig. 3 and Supplementary Table) shows again two metal ions bridged by a rotated D481 side chain. Metal A binds the RNA 3' end and could bind the NTP $\alpha$-phosphate ${ }^{21}$ (Fig. $3 \mathrm{~b}$ and Supplementary Fig. 4). Metal B is $5 \AA$ away from metal A and may move closer for catalysis upon binding NTP $\beta$ - and $\gamma$-phosphates ${ }^{21}$ (Fig. 3b).

The ITC structure indicates that TFIIB stimulates initial RNA synthesis not only by allosteric active-site rearrangement but also by preventing tilting of short DNA-RNA hybrids. Without TFIIB, short hybrids are tilted, and this impairs synthesis, because it occludes the templating DNA base +1 from the active $s^{2} e^{25}$. Such tilting is prevented in the ITC because TFIIB binds the upstream template strand. B-reader residues R64 and D69 contact template strand bases at positions $-7 /-8$ (Fig. 3c). These interactions may also stabilize the initial DNA bubble during open complex formation.

${ }^{1}$ Gene Center and Department of Biochemistry, Center for Integrated Protein Science CIPSM, Ludwig-Maximilians-Universität München, Feodor-Lynen-Str. 25, 81377 Munich, Germany. 
a

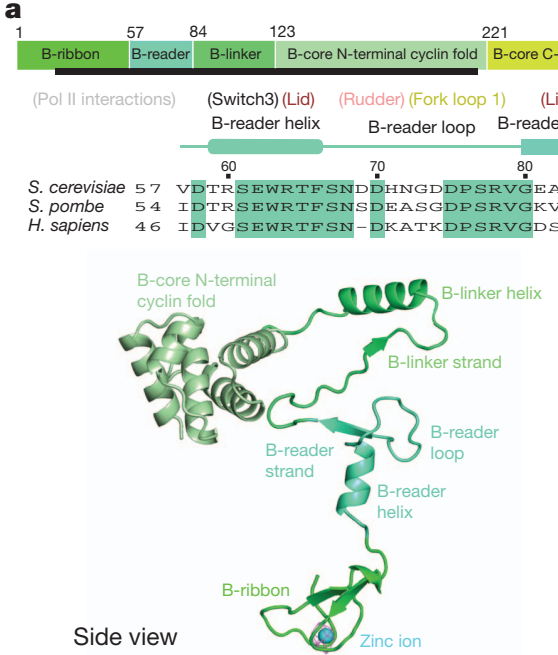

c

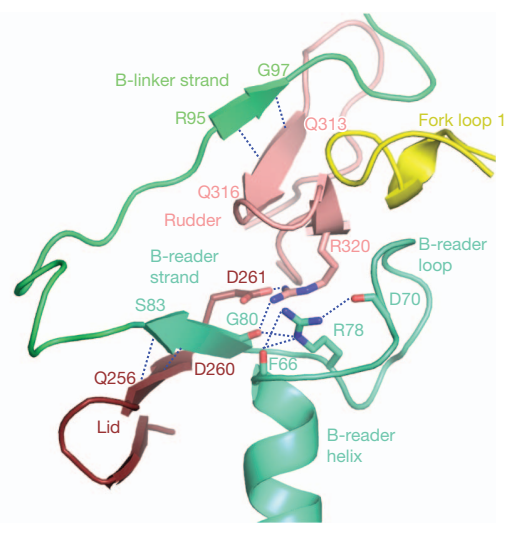

Figure $1 \mid$ Structure of the Pol II-TFIIB complex at 3.4 $\AA$ resolution. a, TFIIB domain organization and ribbon model. The colour code is used throughout the figures. Invariant residues are highlighted. b, View of Pol IITFIIB structure showing the active centre cleft. c, Interactions of B-linker and

a

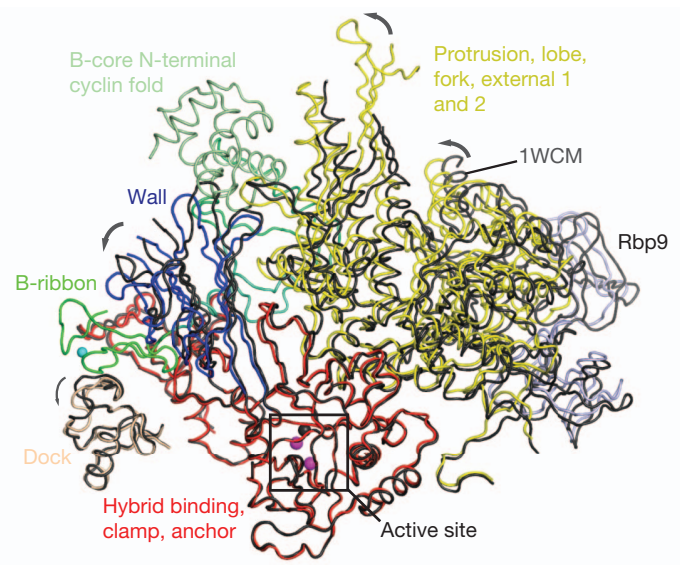

b

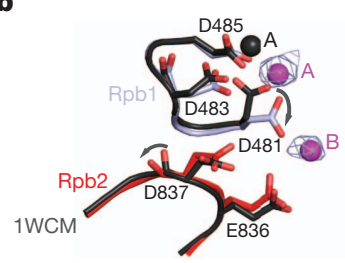

c

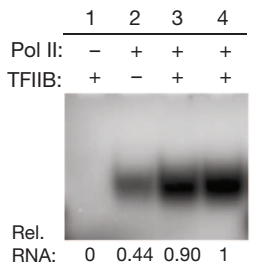

b

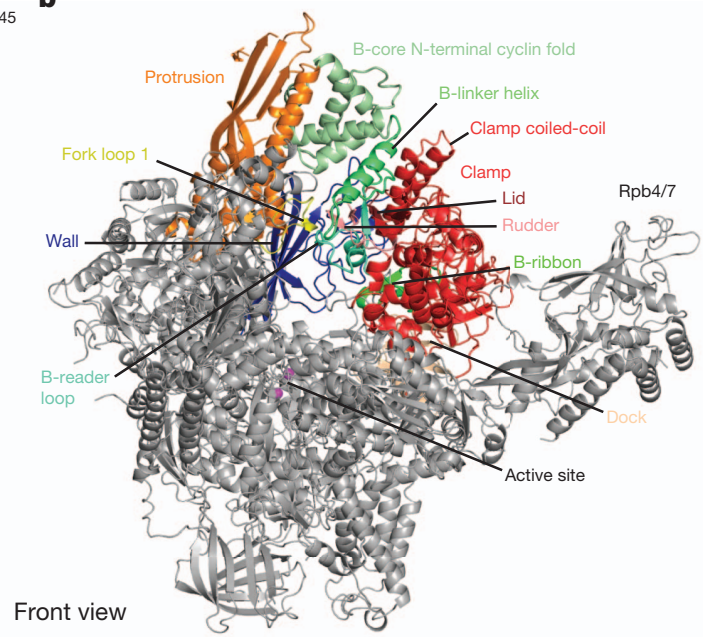

d

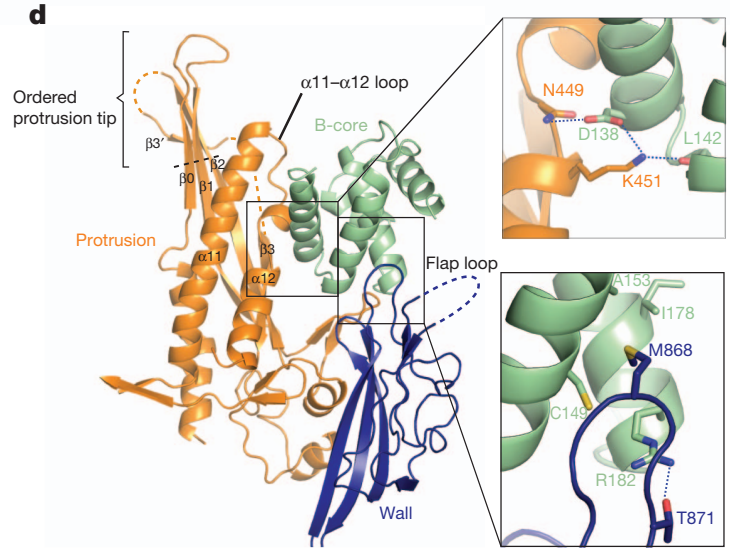

B-reader with Pol II rudder, lid and fork loop 1. d, Interactions of B-core $\mathrm{N}$-terminal cyclin fold with Pol II protrusion and wall. Previous secondary structure nomenclature was maintained ${ }^{19}$ with newly observed elements termed $\beta 0$ (Rpb2 residues 69-74), $\beta 3^{\prime}$ (148-151) and loop $\alpha 11-\alpha 12$ (438-443).

The same contacts contribute to the recognition of an initiator (Inr) DNA sequence that defines the TSS and requires a thymine at template position $-7 /-8$ (refs $1,26,27)$. Interaction of an Inr thymine with the B-reader residue R64 (Fig. 3c) apparently occurs in the open DNA complex, because mutation of R64 causes TSS shifts that are sensitive to changes in Inr sequence ${ }^{27,28}$. The B-reader function in Inr recognition is supported by mutational analysis of the invariant TFIIB residue R78, which buttresses the B-reader loop, including the DNAbinding residue D69 (Fig. 1c). Mutations of R78 shift the TSS in yeast ${ }^{16}$ and human ${ }^{29}$, and can be lethal (Supplementary Fig. 5). Thus, the B-reader adopts a defined structure to assist in TSS selection.

The path of the RNA is blocked by the B-reader loop (Fig. 3). When the RNA grows from 6 to 7 nucleotides, it would clash with three aspartate residues (D70, D74, and D75; Fig. 3c), of which two are conserved among eukaryotes (Fig. 1a). This is consistent with a destabilization of TFIIB binding when RNA grows beyond 6 nucleotides, but TFIIB can be retained beyond this point and is only released when the RNA length reaches $12-13$ nucleotides ${ }^{14}$. Thus, the B-reader loop

Figure $2 \mid$ TFIIB changes Pol II domains and stimulates catalysis. Same colour code as Fig. 1. a, Apparent order of TFIIB domain binding and polymerase domain movements. Pol II-TFIIB structure (colours) and free Pol II ${ }^{10}$ (dark grey) were superimposed with their Rpb1 subunits. b, TFIIB induces active-site rearrangements. Metals A and B are shown as magenta spheres. Difference electron density was contoured at $3.0 \sigma$ (grey mesh, peak height $4.4 \sigma$ (metal A) and $3.6 \sigma$ (metal B)). c, TFIIB stimulates de novo RNA synthesis from a HIS4 promoter-derived tailed template. At least twofold stimulation occurs irrespective of whether TFIIB is added before (lane 3) or after (lane 4) DNA. 
a
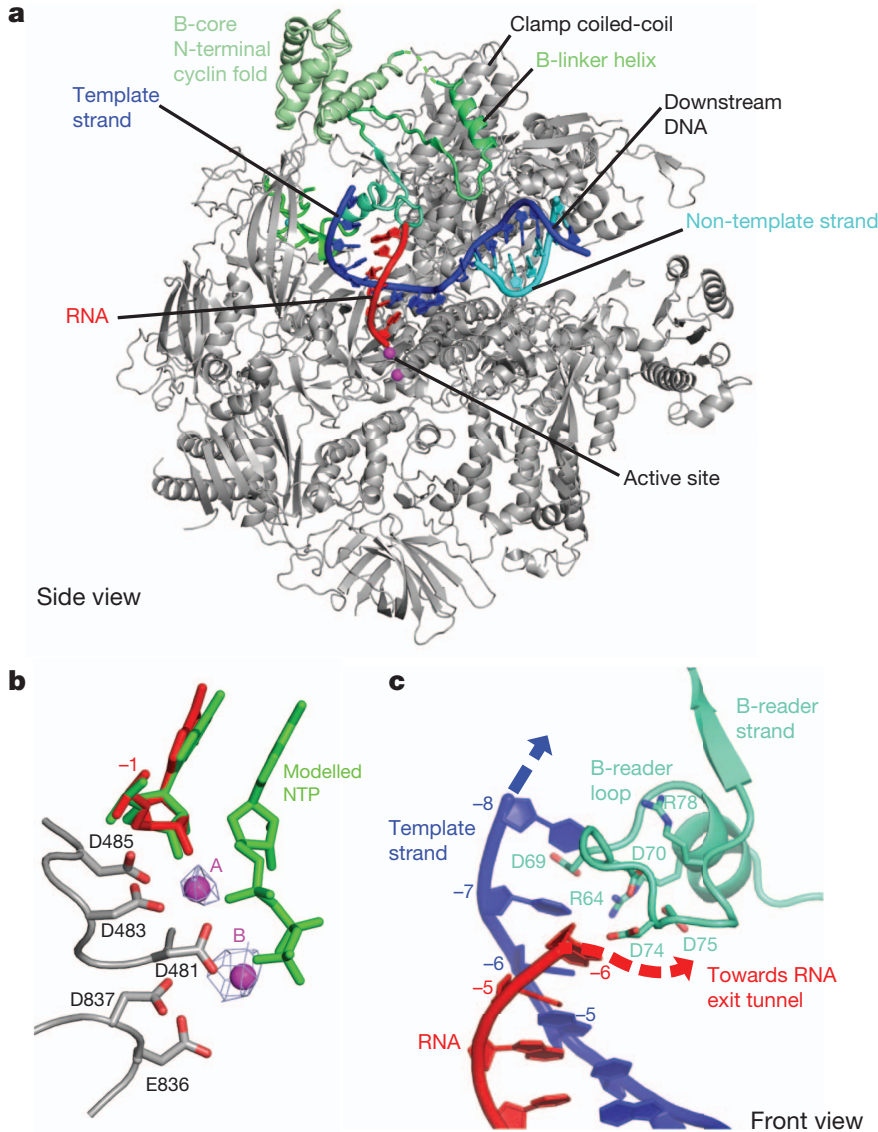

d

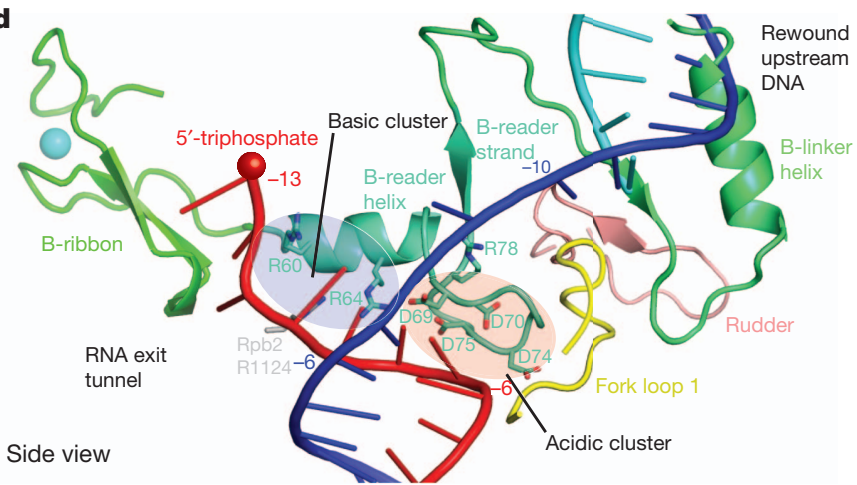

Figure $3 \mid$ Structure of initially transcribing Pol II-TFIIB complex (ITC) elucidates RNA initiation and separation, and TFIIB release. Same colour code as Fig. 1. a, Ribbon model. The view is related to that in Fig. 1a by a $90^{\circ}$ rotation along a vertical axis. b, Active-site configuration with RNA 3'nucleotide in position -1 (red) and superimposed $3^{\prime}$-nucleotide and modelled NTP (green) from a bacterial elongation complex ${ }^{21}$. The metals are covered by difference electron density (grey mesh) contoured at 3.0 $\sigma$ (peak heights $3.8 \sigma$ (metal A) and $4.5 \sigma$ (metal B), respectively). c, B-reader binds the DNA template strand at position $-7 /-8$ and separates the RNA. d, Model of RNA extended into the exit tunnel elucidates TFIIB release.

separates growing RNA from the DNA template and directs it to its exit tunnel. In the elongation complex, RNA separation is achieved by rewinding of the upstream DNA duplex. However, in an ITC with a short RNA that does not yet reach the exit tunnel, such DNA rewinding probably causes collapse of the open DNA bubble, displacing RNA and aborting transcription.

Consistent with a role of the B-reader loop in RNA separation, abortive transcription increases when the loop is destabilized by R78 mutation $^{16}$ (Fig. 1c). B-reader loop function is required for transcription because deletion or mutation of B-reader loop residues 69-75 cause defects in vivo and in vitro (Supplementary Fig. 5), consistent with results for human $\mathrm{TFIIB}^{23}$ and with in vitro data for yeast TFIIB $^{30}$. B-reader loop function is conserved because replacing the loop for its Schizosaccharomyces pombe counterpart preserves function (Supplementary Fig. 5).

We collected diffraction data on ITCs with a 6-nucleotide RNA containing an additional 5'-phosphate and with a 7-nucleotide RNA. The data showed that the B-reader loop was unchanged, consistent with its function in RNA separation. Nucleic acids were present, but partial mobility prevented structure completion. The 6-nucleotide RNA $5^{\prime}$-phosphate was revealed beneath the B-reader loop, and guided modelling of an extended RNA.

The model indicates that RNA separation may be achieved by charge repulsion between the RNA $5^{\prime}$-triphosphate and a cluster of acidic residues in the B-reader loop, and by attraction of the triphosphate to a cluster of basic residues in the exit tunnel (Fig. 3d). Alternatively, the RNA 5'-triphosphate may be trapped by B-reader loop aspartates and a bridging metal ion. Our model accommodates 11-12 nucleotides of RNA. Further RNA extension results in a clash with the B-reader, B-ribbon and wall, explaining why TFIIB is released when the RNA length reaches $12-13$ nucleotides ${ }^{14}$. TFIIB release enables formation of the mature 8-bp DNA-RNA hybrid observed in the elongation complex.

Bacterial RNA polymerase and its initiation factor sigma apparently use similar mechanisms for transcription initiation. The sigma factor is topologically related to $\mathrm{TFIIB}^{1,2}$ and contains the loop region 3.2 which resembles the B-reader loop in location and negative charge ${ }^{6-8}$. Region 3.2 is required for formation of the first RNA phosphodiester bond, normal abortive transcription and sigma factor release $e^{6-8}$.

\section{METHODS SUMMARY}

Saccharomyces cerevisiae 12 -subunit Pol II $\left(3.0 \mathrm{mg} \mathrm{ml}^{-1}\right)$ was incubated with a fivefold molar excess of TFIIB for $30 \mathrm{~min}$ at $298 \mathrm{~K}$ before crystallization by vapour diffusion using 32-34\% (v/v) tacsimate $\mathrm{pH}$ 7.0, $100 \mathrm{mM}$ HEPES $\mathrm{pH} 7.0$, and $5 \mathrm{mM}$ dithiothreitol (DTT) as reservoir solution. Crystals were cryo-protected in mother solution containing $70 \%(\mathrm{v} / \mathrm{v})$ tacsimate, supplemented with $670 \mu \mathrm{M}$ HIS4 tailed template ${ }^{25}$ for ITC complexes, and incubated at $281 \mathrm{~K}$ over night before flashcooling in liquid nitrogen. Diffraction data were collected at $100 \mathrm{~K}$ at SLS beamline X06SA. Structures were solved by molecular replacement (Protein Data Bank code 1WCM). TFIIB was expressed from a pET21 plasmid in Escherichia coli strain Rosetta DE3 (Novagen) and purified by nickel affinity, cation exchange and sizeexclusion chromatography. For in vivo analysis, TFIIB mutants were cloned into vector pRS315 containing $500 \mathrm{bp}$ upstream and $300 \mathrm{bp}$ downstream of the SUA7 open reading frame (ORF), and selected on $5-\mathrm{FOA}$ medium ${ }^{1}$. For in vitro transcription, nuclear extracts were prepared from wild-type and strain SHY245 (ref. 4) as described and assays performed as reported ${ }^{1}$. For the de novo transcription assay, $20 \mu \mathrm{M} \mathrm{rCTP}$, rGTP and $\mathrm{rUTP}$, and $2 \mu \mathrm{M}$ rATP supplemented with $5 \mu \mathrm{Ci}\left[\alpha_{-}{ }^{32} \mathrm{P}\right] \mathrm{rATP}$ were incubated with $2.5 \mathrm{pmol} \mathrm{Pol}$ II, $25 \mathrm{pmol}$ TFIIB and 5 pmol template for $5 \mathrm{~min}$ at $301 \mathrm{~K}$.

Full Methods and any associated references are available in the online version of the paper.

Received 27 July; accepted 26 October 2012.

Published online 14 November 2012.

1. Kostrewa, D. et al. RNA polymerase II-TFIIB structure and mechanism of transcription initiation. Nature 462, 323-330 (2009).

2. Liu, X., Bushnell, D. A., Wang, D., Calero, G. \& Kornberg, R. D. Structure of an RNA polymerase II-TFIIB complex and the transcription initiation mechanism. Science 327, 206-209 (2010)

3. Cho, E. J. \& Buratowski, S. Evidence that transcription factor $\| B$ is required for a post-assembly step in transcription initiation. J. Biol. Chem. 274, 25807-25813 (1999).

4. Ranish, J. A., Yudkovsky, N. \& Hahn, S. Intermediates in formation and activity of the RNA polymerase II preinitiation complex: holoenzyme recruitment and a postrecruitment role for the TATA box and TFIIB. Genes Dev. 13, 49-63 (1999).

5. Vannini, A. \& Cramer, P. Conservation between the RNA polymerase I, II, and III transcription initiation machineries. Mol. Cell 45, 439-446 (2012).

6. Vassylyev, D. G. et al. Crystal structure of a bacterial RNA polymerase holoenzyme at 2.6A resolution. Nature 417, 712-719 (2002).

7. Murakami, K. S., Masuda, S. \& Darst, S. A. Structural basis of transcription initiation: RNA polymerase holoenzyme at 4 A resolution. Science 296, 1280-1284 (2002) 
8. Kulbachinskiy, A. \& Mustaev, A. Region 3.2 of the sigma subunit contributes to the binding of the 3 '-initiating nucleotide in the RNA polymerase active center and facilitates promoter clearance during initiation. J. Biol. Chem. 281, 18273-18276 (2006).

9. Naji, S., Bertero, M. G., Spitalny, P., Cramer, P. \& Thomm, M. Structure-function analysis of the RNA polymerase cleft loops elucidates initial transcription, DNA unwinding and RNA displacement. Nucleic Acids Res. 36, 676-687 (2008).

10. Armache, K. J., Mitterweger, S., Meinhart, A. \& Cramer, P. Structures of complete RNA polymerase II and its subcomplex, Rpb4/7. J. Biol. Chem. 280, 7131-7134 (2005).

11. Grünberg, S., Warfield, L. \& Hahn, S. Architecture of the RNA polymerase II preinitiation complex and mechanism of ATP-dependent promoter opening. Nature Struct. Mol. Biol. 19, 788-796 (2012).

12. Chen, Z. A. et al. Architecture of the RNA polymerase II-TFIIF complex revealed by cross-linking and mass spectrometry. EMBO J. 29, 717-726 (2010).

13. Eichner, J., Chen, H. T., Warfield, L. \& Hahn, S. Position of the general transcription factor TFIIF within the RNA polymerase II transcription preinitiation complex. EMBO J. 29, 706-716 (2010).

14. Cabart, P., Ujvari, A., Pal, M. \& Luse, D. S. Transcription factor TFIIF is not required for initiation by RNA polymerase II, but it is essential to stabilize transcription facto TFIIB in early elongation complexes. Proc. Natl Acad. Sci. USA 108, 15786-15791 (2011).

15. Fishburn, J. \& Hahn, S. Architecture of the yeast RNA polymerase II open complex and regulation of activity by TFIIF. Mol. Cell. Biol. 32, 12-25 (2012).

16. Chen, B. S. \& Hampsey, M. Functional interaction between TFIIB and the Rpb2 subunit of RNA polymerase II: implications for the mechanism of transcription initiation. Mol. Cell. Biol. 24, 3983-3991 (2004).

17. Steitz, T. A., Smerdon, S. J., Jager, J. \& Joyce, C. M. A unified polymerase mechanism for nonhomologous DNA and RNA polymerases. Science 266,2022-2025 (1994).

18. Sosunov, V. etal. Unified two-metal mechanism of RNA synthesis and degradation by RNA polymerase. EMBO J. 22, 2234-2244 (2003).

19. Cramer,P., Bushnell, D. A. \& Kornberg, R. D. Structural basis of transcription: RNA polymerase II at 2.8 angstrom resolution. Science 292, 1863-1876 (2001).

20. Wang, D., Bushnell, D. A., Westover, K. D., Kaplan, C. D. \& Kornberg, R. D. Structural basis of transcription: role of the trigger loop in substrate specificity and catalysis. Cell 127, 941-954 (2006).

21. Vassylyev, D. G. et al. Structural basis for substrate loading in bacterial RNA polymerase. Nature 448, 163-168 (2007).

22. Werner, F. \& Weinzierl, R. O. Direct modulation of RNA polymerase core functions by basal transcription factors. Mol. Cell. Biol. 25, 8344-8355 (2005).

23. Tran, K. \& Gralla, J. D. Control of the timing of promoter escape and RNA catalysis by the transcription factor Ilb fingertip. J. Biol. Chem. 283, 15665-15671 (2008)
24. Pal, M.,Ponticelli, A. S. \& Luse, D. S. The role of the transcription bubble and TFIIB in promoter clearance by RNA polymerase II. Mol. Cell 19, 101-110 (2005).

25. Cheung, A. C., Sainsbury, S. \& Cramer, P. Structural basis of initial RNA polymerase II transcription. EMBO J. 30, 4755-4763 (2011).

26. Zhang, Z. \& Dietrich, F. S. Mapping of transcription start sites in Saccharomyces cerevisiae using 5' SAGE. Nucleic Acids Res. 33, 2838-2851 (2005).

27. Kuehner, J. N. \& Brow, D. A. Quantitative analysis of in vivo initiator selection by yeast RNA polymerase II supports a scanning model. J. Biol. Chem. 281, 14119-14128 (2006).

28. Bangur, C. S., Pardee, T. S. \& Ponticelli, A. S. Mutational analysis of the D1/E1 core helices and the conserved $\mathrm{N}$-terminal region of yeast transcription factor IIB (TFIIB): identification of an N-terminal mutant that stabilizes TATA-binding protein-TFIIB-DNA complexes. Mol. Cell. Biol. 17, 6784-6793 (1997).

29. Hawkes, N. A. \& Roberts, S. G. The role of human TFIIB in transcription start site selection in vitro and in vivo. J. Biol. Chem. 274, 14337-14343 (1999).

30. Yang, C. \& Ponticelli, A. S. Evidence that RNA polymerase II and not TFIIB is responsible for the difference in transcription initiation patterns between Saccharomyces cerevisiae and Schizosaccharomyces pombe. Nucleic Acids Res. 40, 6495-6507 (2012)

Supplementary Information is available in the online version of the paper.

Acknowledgements We thank C. Bäjen, A. Cheung, S. Etzold, F. Hög, D. Kostrewa, N. Pirkl and other members of the Cramer laboratory. Part of this work was performed at the Swiss Light Source (SLS) at the Paul Scherrer Institut, Villigen, Switzerland. S.S. was supported by a postdoctoral fellowship from the Alexander-von-Humboldt Foundation. P.C. was supported by the Deutsche Forschungsgemeinschaft, SFB646, TR5, GraKo1721, SFB960, CIPSM, NIM, an Advanced Grant of the European Research Council, the LMUinnovativ project Bioimaging Network, the Jung-Stiftung, and the Vallee Foundation.

Author Contributions S.S. carried out experiments except for functional analysis presented in Supplementary Fig. 5, which was carried out by J.N. P.C. initiated and supervised the project. S.S. and P.C. prepared the manuscript.

Author Information Coordinates and structure factors of the Pol II-TFIIB complex and the ITC with 6-nucleotide RNA have been deposited in the Protein Data Bank under accession numbers $4 B B R$ and $4 B B S$, respectively. Reprints and permissions information is available at www.nature.com/reprints. The authors declare no competing financial interests. Readers are welcome to comment on the online version of the paper. Correspondence and requests for materials should be addressed to P.C. (cramer@Imb.uni-muenchen.de). 


\section{METHODS}

Preparation of $\boldsymbol{S}$. cerevisiae Pol II and recombinant TFIIB. Saccharomyces cerevisiae 12-subunit Pol II was prepared as described ${ }^{31}$. TFIIB (residues 19215) was cloned into pET21b with a C-terminal $\mathrm{His}_{6}$ tag and expressed in E. coli Rosetta (DE3) (Novagen). The culture was grown in LB medium at $37^{\circ} \mathrm{C}$ until absorbance at $600 \mathrm{~nm}$ of $0.6,0.5 \mathrm{mM}$ IPTG was added and the culture was grown for a further $18 \mathrm{~h}$ at $20^{\circ} \mathrm{C}$. Cells were collected by centrifugation and frozen in liquid nitrogen. Protein was purified by nickel affinity, cation exchange and sizeexclusion chromatography. Cells were lysed by sonication in buffer A ( $50 \mathrm{mM}$ Tris $\mathrm{pH} 7.5,300 \mathrm{mM} \mathrm{NaCl}, 10 \mu \mathrm{M} \mathrm{ZnCl}_{2}, 2 \mathrm{mM}$ dithiothreitol (DTT) supplemented with $5 \mathrm{mM}$ imidazole, $0.2 \%(\mathrm{v} / \mathrm{v})$ Tween 20 and $1 \times$ protease inhibitors $(100 \times$ stock: $1.42 \mathrm{mg}$ leupeptin, $6.85 \mathrm{mg}$ pepstatin A, $850 \mathrm{mg}$ PMSF, 1,650 mg benzamidine in $50 \mathrm{ml}$ ethanol)). After centrifugation at $30,000 \mathrm{~g}$ for $30 \mathrm{~min}$, the cleared lysate was applied to a pre-equilibrated (buffer A) Ni-NTA agarose column (Qiagen). The column was washed with 10 column volumes of buffer A containing $10 \mathrm{mM}$ imidazole before elution of the protein with buffer A containing $200 \mathrm{mM}$ imidazole. The conductivity of the eluate was adjusted to match that of buffer B ( $50 \mathrm{mM}$ Tris $\mathrm{pH} 7.5$, $100 \mathrm{mM} \mathrm{NaCl}, 2 \mathrm{mM}$ DTT) and applied to a MonoS 10/100 GL column (GE healthcare) equilibrated in buffer $\mathrm{B}$. The protein was eluted with a linear gradient from $100 \mathrm{mM}$ to $1 \mathrm{M} \mathrm{NaCl}$. To remove any minor contaminants a final size exclusion step using a Superdex 75 10/300 GL column (GE Healthcare) in $5 \mathrm{mM}$ NaHEPES (pH 7.25 at $20{ }^{\circ} \mathrm{C}$ ), $40 \mathrm{mM}\left(\mathrm{NH}_{4}\right)_{2} \mathrm{SO}_{4}, 10 \mu \mathrm{M} \mathrm{ZnCl}_{2}, 10 \mathrm{mM}$ DTT was carried out. The protein was concentrated to $3 \mathrm{mg} \mathrm{ml}^{-1}$ in an Amicon Ultra4 Centrifugal Filter Unit (Millipore) and flash-frozen in liquid nitrogen. TFIIB reader variants were cloned with a $\mathrm{C}$-terminal $\mathrm{His}_{6}$-tag into $\mathrm{pOPINE}^{32}$ (provided by OPPFUK) using In-Fusion HD cloning kit (Contech) and purified as described above. Yeast strains and promoter-dependent in vitro transcription assay. Mutations in TFIIB were introduced by PCR. Both wild-type and mutated TFIIB were cloned into pRS3 $35^{33}$ containing $500 \mathrm{bp}$ upstream and $300 \mathrm{bp}$ downstream of the SUA7 ORF. After transformation into the SUA7 shuffle strain ${ }^{1}$, selection and growth characterization of the respective TFIIB variants was carried out on 5-FOA medium as described ${ }^{1}$. All growth experiments were made as triplicates. Nuclear extracts were prepared from wild-type and strain SHY245 (ref. 4) as reported previously $^{34}$. In vitro transcription was essentially performed as described ${ }^{34}$, with minor modifications. As template, $150 \mathrm{ng}$ of a plasmid encoding for a HIS4-SNR14 (pMSe58) fusion promoter was used and the transcription buffer contained $20 \mathrm{mM}$ HEPES $\mathrm{pH}$ 7.6, $100 \mathrm{mM}$ potassium acetate, $1 \mathrm{mM}$ EDTA, $5 \mathrm{mM}$ magnesium acetate and $2.5 \mathrm{mM}$ DTT. For activated transcription, $150 \mathrm{ng}$ of the recombinant Gal4-VP16 fusion protein and $10 \mathrm{pmol}$ of the respective recombinant TFIIB variant were used. Primer extension was carried out using $0.125 \mathrm{pmol}$ of a fluorescently labelled DNA primer (5'-Cy5-TTCACCAGTGAGACGGGC $\mathrm{AAC})$. The transcripts were separated using a $6 \%$ polyacrylamide/7 $\mathrm{M}$ urea TBE gel, scanned with a Typhoon 9410 and quantified with the ImageQuant software (GE healthcare).

Promoter-independent de novo transcription assay with $3^{\prime}$-tailed DNA templates. $10 \mu \mathrm{l}$ reactions of $2.5 \mathrm{pmol}$ Pol II, $25 \mathrm{pmol}$ TFIIB and 5 pmol HIS $43^{\prime}$ tailedtemplate in $20 \mathrm{mM}$ HEPES pH 7.6, $60 \mathrm{mM}\left(\mathrm{NH}_{4}\right)_{2} \mathrm{SO}_{4}, 2 \mathrm{mM} \mathrm{MgSO}_{4}, 10 \%(\mathrm{v} / \mathrm{v})$ glycerol, $10 \mu \mathrm{M} \mathrm{ZnCl}_{2}$, were incubated at $25^{\circ} \mathrm{C}$ for a total of $30 \mathrm{~min}$. Pol II was added first and there was a short 5 -min incubation between the addition of the template and TFIIB. After addition of $20 \mu \mathrm{M}$ rCTP, rGTP and rUTP, and $2 \mu \mathrm{M}$ rATP (Fig. 2c) or $500 \mu \mathrm{M}$ rCTP, rGTP and rUTP, and $50 \mu \mathrm{M}$ rATP (Supplementary Fig. 3) supplemented with $5 \mu \mathrm{Ci}\left[\alpha_{-}{ }^{32} \mathrm{P}\right]$ rATP (Hartmann Analytic) the reactions were incubated at $28^{\circ} \mathrm{C}$ for $5 \mathrm{~min}$. The run-off products were separated on a $20 \%$ denaturing polyacrylamide gel and visualized by phosphorimaging by a Storm 860 scanner (Molecular Dynamics). Relative RNA levels were quantified with ImageQuant TL (GE Healthcare).

31. Sydow, J. F. et al. Structural basis of transcription: mismatch-specific fidelity mechanisms and paused RNA polymerase II with frayed RNA. Mol. Cell 34, 710-721 (2009).

32. Berrow, N. S. et al. A versatile ligation-independent cloning method suitable for high-throughput expression screening applications. Nucleic Acids Res. 35, e45 (2007).

33. Sikorski, R. S. \& Hieter, P. A system of shuttle vectors and yeast host strains designed for efficient manipulation of DNA in Saccharomyces cerevisiae. Genetics 122, 19-27 (1989).

34. Seizl, M., Lariviere, L., Pfaffeneder, T., Wenzeck, L. \& Cramer, P. Mediator head subcomplex Med11/22 contains a common helix bundle building block with a specific function in transcription initiation complex stabilization. Nucleic Acids Res. 39, 6291-6304 (2011). 